Madrid, Spain; ${ }^{8}$ Hospital de Albacete, Rheumatology, Albacete, Spain; ${ }^{9}$ Hospital de Basurto, Bilbao, Spain; ${ }^{10}$ Hospital Virgen de las Nieves, Rheumatology, Granada, Spain

Objectives: To investigate the prevalence, risk factors, and effects of primary renal disease on morbidity and mortality in patients with primary Sjögren's syndrome (pSS).

Methods: All patients in the SJÖGRENSER (registry of adult SSp patients of the Spanish Society of Rheumatology, cross-sectional phase) cohort were retrospectively investigated for the presence of clinically significant renal involvement directly related to $\mathrm{pSS}$ activity.

Results: Of the 437 patients investigated, 39 (9\%) presented overt renal involvement during follow-up. Severe renal disease necessitating kidney biopsy was relatively rare $(23 \%)$.

Renal involvement may complicate pSS at any time during the disease course and is associated with severe disease (indicated by higher scores of involvement, activity, and damage), systemic multiorgan involvement, and a higher frequency of lymphoma. Multivariate analysis showed that older age (odds ratio [OR] 1.03, 95\% confidence interval [Cl] 1.00-1.07), higher European League Against Rheumatism Sjogren's Syndrome Disease Activity Index scores (OR 1.1, $\mathrm{Cl} 1.03-1.18$ ), serum anti-La/SSB positivity (OR 6.44, $\mathrm{Cl} 1.36-30.37$ ), and non-vasculitic cutaneous involvement (OR 8.64, 1.33-55.90) were independently associated with this complication.

Chronic renal failure developed in 23 of 39 patients (59\%); only 1 of them progressed to end-stage renal disease necessitating renal replacement therapy. Patients with renal disease showed higher Sjögren's syndrome disease damage index scores (SSDDI), higher rates of hospitalization due to disease activity and higher rates of clinically relevant comorbidities.

Conclusion: Renal involvement is an uncommon complication in pSS that was observed in $9 \%$ of patients. Although categorized as a non-negligible comorbidity, this condition shows a favorable prognosis.

Disclosure of Interests: None declared

DOI: 10.1136/annrheumdis-2020-eular.5149

\section{FRI0183 DISTINCTIVE TRAITS OF MYOCARDIAL INFLAMMATION IN PATIENTS WITH SYSTEMIC LUPUS ERYTHEMATOSUS: A MULTICENTRE STUDY}

G. A. Ramirez ${ }^{1,2}$, M. Gerosa ${ }^{3,4}$, G. De Luca ${ }^{1,2}$, L. Beretta ${ }^{5}$, S. Sala ${ }^{6}$, G. Peretto ${ }^{6}$, L. Moroni ${ }^{1,2}$, F. Mastropaolo ${ }^{1,2}$, A. Cariddi ${ }^{1}$, S. Sartorelli ${ }^{1}$, C. Campochiaro ${ }^{1,2}$, E. Bozzolo ${ }^{1}$, R. Caporali ${ }^{3,4}$, L. Dagna ${ }^{1,2}$ on behalf of Milan SLE Consortium (SMiLE). ${ }^{1}$ IRCCS H San Raffaele, UnIRAR, Milan, Italy; ${ }^{2}$ Università Vita-Salute San Raffaele, Milan, Italy; ${ }^{3}$ ASST Gaetano Pini -CTO, Clin. Rheumatology Unit, Milan, Italy; ${ }^{4}$ University of Milan, Dep. of Clin. Science of Community Health, Milan, Italy; ${ }^{5}$ Fondazione IRCCS Ca' Granda H Maggiore Policlinico, Referral Center for Systemic Autoimmune Diseases, Milan, Italy; ${ }^{6}$ RCCS H San Raffaele, Unit of Cardiology, Milan, Italy

Background: Myocarditis is an infrequent but potentially life-threatening inflammatory disorder and might be part of the spectrum of systemic lupus erythematosus (SLE). Little is known about the clinical and histologic features of myocarditis in SLE, especially compared to other forms of myocarditis.

Objectives: to test for potential distinctive traits among myocarditis in SLE (MyoSLE), SLE without myocarditis (OnlySLE) and myocarditis without SLE (OnlyMyo)

Methods: Patients with MyoSLE were identified from three centres and compared with 231 cross-sectionally enrolled patients with OnlySLE and 87 patients with OnlyMyo. MyoSLE patients were split into two groups based on myocarditis onset within (early onset) vs after (late onset) the first year from SLE diagnosis. OnlySLE patients were dichotomised in the same way based on disease duration at time of enrolment. Demographics and general clinical features were collected retrospectively. SLE disease activity index 2000 (SLEDAI-2K), SLE International Collaborating Clinics/American College of Rheumatology damage index (SDI), clinical and laboratory features were collected at time of myocarditis onset in MyoSLE and at enrolment in OnlySLE. Quantitative data are expressed as median [interquartile range].

Results: Fourteen MyoSLE patients were identified, $50 \%$ with early onset. Women were equally frequent among MyoSLE (71\%) and OnlySLE patients $(87 \%)$ and less frequent in the OnlyMyo group (43\%; $p<0.001)$. Age was comparable among groups. Clinical features at presentation, including left ventricular ejection fraction, were similar between MyoSLE and OnlyMyo, although the former had higher levels of pro-brain natriuretic peptide (1.1 [0.4-1.8] vs 0.1 [0.1$0.5] \mathrm{ng} / \mathrm{ml} ; \mathrm{p}=0.004)$. Patients with MyoSLE also had a lower frequency of left ventricle lateral wall involvement ( 36 vs $68 \% ; p=0.035$ ) and of oedema (20 vs $71 \% ; p=0.036$ ) and necrosis (0 vs $64 \% ; p=0.009$ ) at biopsy. Antiphospholipid antibodies (aPL) were more frequent in MyoSLE (57\%) compared to both OnlyMyo $(16 \% ; p=0.003)$ and OnlySLE $(28 \% ; p=0.031)$. Compared to OnlySLE, patients with MyoSLE also had a higher prevalence of aPL-syndrome (APS: 36 vs $7 \% ; p=0.003$ ), neuropsychiatric (NPSLE: 43 vs $19 \% ; p=0.039$ ) and gastrointestinal manifestations ( $21 \mathrm{vs} 5 \% ; p=0.045$ ). Early and late onset patients had similar demographics and clinical features and did not differ from patients with OnlySLE with similar disease duration in terms of SLEDAI-2K and SDI. Late onset MyoSLE patients had a higher prevalence of NPSLE (57 vs $18 \%$; $p=0.026)$ and APS ( 57 vs $7 \% ; p=0.001)$ and higher $C$-reactive protein levels ( 6 [2-12] vs $1[0-4] \mathrm{mg} / \mathrm{l} ; \mathrm{p}=0.024)$ compared to OnlySLE patients with the same disease duration.

Conclusion: Demographics of patients with MyoSLE are more similar to patients with OnlySLE than to OnlyMyo patients. MyoSLE might have distinct histological and pathogenic features compared to OnlyMyo. Patients with MyoSLE show similar patterns of disease activity and accrued damage at time of myocarditis onset compared to patients with OnlySLE with the same disease duration but might diverge later on in SLE course. aPL are frequent in MyoSLE and might both contribute to the pathogenesis of myocardial inflammation and account for the high prevalence of NPSLE and APS, especially in late onset cases.

\section{References:}

[1] Gartshteyn $Y$ et al., Lupus, 2020

[2] Thomas G et al., J Rheumatol, 2017

[3] Peretto G et al., Int J Cardiol, 2019

[4] McDonnell T et al., Blood Rev, 2019

Disclosure of Interests: Giuseppe Alvise Ramirez: None declared, Maria Gerosa: None declared, Giacomo De Luca Speakers bureau: SOBI, Novartis Celgene, Pfizer, MSD, Lorenzo Beretta Grant/research support from: Pfizer, Simone Sala: None declared, Giovanni Peretto: None declared, Luca Moroni: None declared, Francesca Mastropaolo: None declared, adriana cariddi: None declared, Silvia Sartorelli: None declared, Corrado Campochiaro Speakers bureau: Novartis, Pfizer, Roche, GSK, SOBI, Enrica Bozzolo: None declared, Roberto Caporali Consultant of: AbbVie; Gilead Sciences, Inc.; Lilly; Merck Sharp \& Dohme; Celgene; Bristol-Myers Squibb; Pfizer; UCB, Speakers bureau: Abbvie; Bristol-Myers Squibb; Celgene; Lilly; Gilead Sciences, Inc; MSD; Pfizer; Roche; UCB, Lorenzo Dagna Grant/research support from: The Unit of Immunology, Rheumatology, Allergy and Rare Diseases (UnIRAR) received unresctricted research/educational grants from Abbvie, Bristol-Myers Squibb, Celgene, Janssen, Merk Sharp \& Dohme, Mundipharma Pharmaceuticals, Novartis, Pfizer, Roche, Sanofi-Genzyme, and SOBI., Consultant of: Prof Lorenzo Dagna received consultation honoraria from Abbvie, Amgen Biogen, Bristol-Myers Squibb, Celltrion, Novartis, Pfizer, Roche, Sanofi-Genzyme, and SOBI.

DOI: 10.1136/annrheumdis-2020-eular.4653

\section{FRI0184 \\ ATTRIBUTION OF NEUROPSYCHIATRIC MANIFESTATIONS TO SYSTEMIC LUPUS ERYTHEMATOSUS IN POLISH COHORT OF PATIENTS WITH THE USE OF THE ITALIAN MODEL}

K. Pawlak-Bus ${ }^{1,2}$, W. Schmidt ${ }^{1,2}$, P. Leszczynski ${ }^{1,2} .{ }^{1}$ Poznan University of Medical Sciences, Department of Rheumatology and Rehabilitation, Poznan, Poland; ' Jozef Strus Municipal Hospital, Department of Rheumatology and Osteoporosis, Poznan, Poland

Background: Distinguishing primary NPSLE (neuropsychiatric systemic lupus erythematosus) from secondary causes remains challenging (1). Attribution models were developed in order to aim clinicians in correct classification of NPSLE cases (2).

Objectives: To investigate the prevalence of primary NPSLE manifestations assigned with Italian model of attribution (2).

Methods: We retrospectively assessed clinical details of 164 patients with SLE classified with 2012 SLICC (Systemic Lupus International Collaborating Clinics) classification criteria, 21 were excluded due to incomplete information. Data was gathered with a questionnaire comprising demographics, medical history, laboratory results (concentrations of antibodies against double stranded DNA - anti-dsDNA, complement components C3 and C4), disease activity measured with Systemic Lupus Erythematosus Disease Activity Index 2000 (SLEDAI-2K) and Physician Global Assessment (PGA) and damage determined with SLICC/ ACR (American College of Rheumatology) Damage Index (SDI). Neuropsychiatric manifestations were categorized in accordance with 1999 ACR glossary and attribution of manifestations was performed with the use of Italian model with the score $\geq 7$ out of 10 points enabling assignment to primary NPSLE group (2). Statistical analysis was conducted with Statistica v.13.3 using Mann-Whitney U, chi-square and Fisher exact test.

Results: We encountered 155 NP manifestations in our cohort and 52 (34\%) were attributed to SLE. Characteristics of the study groups are presented in Table 1. Exact manifestations and their attribution rates are presented on 